\title{
Cylindrical Aggregates of 5,5',6,6'-Tetrachlorobenzimida- carbocyanine Amphiphilic Derivatives: Structure-Related Optical Properties and Exciton Dynamics
}

\author{
A. Pugzlys, P. R. Hania, R. Augulis, K. Duppen, and P. H. M. van Loosdrecht \\ Materials Science Centre, University of Groningen, Nijenborgh 4, 9747 AG Groningen, The Netherlands
}

Received 22 May 2006; Revised 26 June 2006; Accepted 28 July 2006

The static and time-resolved optical response of 5,5',6,6'-tetrachlorobenzimidacarbocyanine chromophore-based tubular double-wall molecular aggregates is studied. The linear dichroism and absorption spectra have the same basic structure for all aggregates investigated essentially showing a set of narrow and highly polarized bands originating from inner and outer wall excitations. The exact positions and strengths of absorption bands are influenced by the choice of side groups and various additives. Time-resolved fluorescence measurements show a substantial speeding of the fluorescence decay upon aggregation, mainly resulting from collective effects. Pump-probe experiments evidence slow inner-to-outer and fast outer-to-inner wall energy transfers between the walls of the tubules.

Copyright (C) 2006 A. Pugzlys et al. This is an open access article distributed under the Creative Commons Attribution License, which permits unrestricted use, distribution, and reproduction in any medium, provided the original work is properly cited.

\section{INTRODUCTION}

J-aggregates of organic dyes discovered in the mid thirties by Scheibe $[1,2]$ and, independently, by Jelley $[3,4]$ posses unique linear and nonlinear optical properties, which originate from their highly delocalized $\pi$-electron systems. Nowadays these molecular dye aggregates play an important role in many technological applications. Because of their large light absorption cross-section these aggregates are used as spectral sensitizers in photographic industry [5] and have a large potential for use in the field of opto-electronics and nonlinear optics $[6,7]$. In addition, because of highly efficient excitation energy collection and transfer, molecular aggregates may also be considered as a model system for light harvesting complexes and energy transport units in artificial photosynthesis. Natural photosynthetic systems are composed of light harvesting units based on aggregates of chlorophylls and carotenoids and possess a variety of morphologies [8-10].

In order to render molecular aggregates useful in sophisticated artificial supramolecular transport systems, control over the aggregate morphology is required. This can be done via modification of amphiphilic properties arising from the hydrophobic (apolar) and hydrophilic (polar or ionic) substituents of the dye molecules. Amphiphiles form aggregates in water above a certain minimum concentration. It was recently shown in the group of Dähne
$[11,12]$ that such modifications of the cyanine dye $5,5^{\prime}, 6,6^{\prime}-$ tetrachlorobenzimidacarbocyanine (TBC, chemical structure is shown in Figure 1) indeed allow control over the morphology of the formed aggregates. Self-assembly of various TBC aggregates is driven by the hydrophobic forces and hydrogen bonding determined by the hydrophobic and hydrophilic $1,1^{\prime}$ and $3,3^{\prime}$ nitrogen substituents, and in addition by the dispersion forces between the highly delocalized $\pi$ electron systems and the electrostatic forces due to the delocalized positive charge of the chromophore. Although the electronic structure of a single chromophore, and therefore its optical properties, remain unchanged [13], the nature of $1,1^{\prime}$ and $3,3^{\prime}$ nitrogen substituents substantially influences both morphology and optical properties of the aggregates. For instance in the case of 1,1' -diethyl, 3,3'-bis (4-sulfobutyl) substituents of the TBC chromophore linear (quasi-onedimensional) aggregates are formed [14]. A sharp and intense band (J-band) centered at $587 \mathrm{~nm}$ dominates the linear absorption spectrum of these aggregates. In contrast, stacks of bilayer ribbons are formed in the case of $1,1^{\prime}$ dioctyl and 3,3'-bis(4-carboxybutyl) substituents while a slight change in the 3,3' nitrogen substituents from bis(4-carboxybutyl) to bis(3-carboxypropyl) (C8O3, see Figure 1) leads to a selfarrangement into superhelical assemblies of tubular strands $[12,13]$. When instead bis(3-sulfopropyl) (C8S3) is used in the substituents, isolated double wall tubules are formed. 


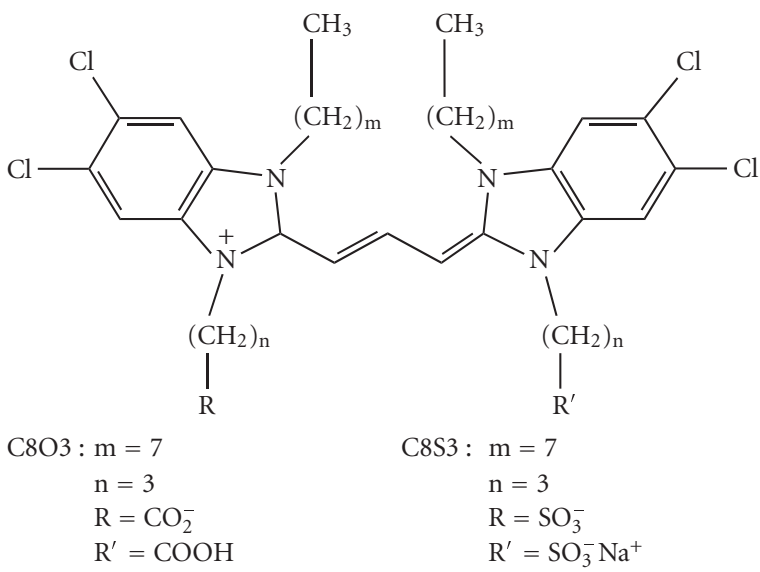

Figure 1: Molecular structure of 5,5',6,6' -tetrachlorobenzimidacarbocyanine (TBC) chromophore. The lower part lists $1,1^{\prime}$, and $3,3^{\prime}$ nitrogen substituents relevant to $\mathrm{C} 8 \mathrm{O} 3$ and $\mathrm{C} 8 \mathrm{~S} 3$.

Formation of cylindrical aggregates leads to the appearance of multiple bands in the linear absorption spectrum, which are red shifted with respect to the monomer absorption and correspond to transitions with differently oriented transition dipole moments [15]. In addition to the choice of side groups, slight changes in local environment, induced by addition of various surfactants, also cause substantial changes in the morphology [15-18].

In addition to the large amount of experimental data on optical and structural properties of the $\mathrm{C} 8 \mathrm{O} 3$-based cylindrical aggregates, recently there has also been substantial theoretical and numerical progress in explaining the complicated absorption spectra of double-wall cylindrical C8S3 aggregates [19]. Each wall of the double-wall cylinder can be modeled as a brick-layer lattice wrapped to a cylindrical surface with each unit cell of the lattice occupied by single C8S3 molecule. The collective excitations of the aggregates, resulting from intermolecular dipole-dipole interactions, may be described within a Frenkel exciton model. The model leads to two dominant exciton bands for each cylinder, one polarized parallel to the cylinder's axis and one perpendicular to it. Apart from a successful interpretation of experimental observations, the simulations allowed determination of microscopic structural parameters, that is, the geometry of packing of C8S3 molecules in the aggregate. However the model could not completely explain the structure and optical properties of $\mathrm{C} 8 \mathrm{O} 3$ aggregates, mainly because of an additional band located on the blue side of the spectrum and governed by transitions having dipole moments aligned parallel to the aggregate axis.

In this paper we summarize some of our previous results and extend these with a study of morphology-related optical properties of $\mathrm{C} 8 \mathrm{O} 3$ aggregates by analyzing modification of the optical response of $\mathrm{C} 8 \mathrm{O} 3$ aggregates upon using different additives. In addition we report on a study of the exciton transport between the inner and outer walls of $\mathrm{C} 8 \mathrm{O} 3$ cylindrical aggregates using time-resolved fluores- cence and frequency-resolved polarization selective pumpprobe experiments.

\section{OPTICAL PROPERTIES OF CYLINDRICAL AGGREGATES}

We start the discussion on optical properties of TBC-based cylindrical aggregates with a summary of structural modifications which appear in the presence of different additives. According to cryo-TEM C8S3 aggregates represent separate double-wall tubules of thickness $15-16 \mathrm{~nm}$ and lengths up to several micrometers [19] while $\mathrm{C} 8 \mathrm{O} 3$ aggregates are linear rope-like assemblies of slightly thinner $(10-12 \mathrm{~nm})$ tubular strands with a total thickness in the order of a few tens of nanometers [12]. Addition of short chain alcohols to a water solution of $\mathrm{C} 8 \mathrm{O} 3$ aggregates induces formation of much thicker and longer superhelices as has been observed via cryo-TEM measurements [18]. These superhelices are an order of magnitude thicker than the pure $\mathrm{C} 8 \mathrm{O} 3$ aggregates. In addition, the diameter of a single tubule is slightly increased from $10 \pm 0.5 \mathrm{~nm}$ in the case of pure $\mathrm{C} 8 \mathrm{O} 3$ aggregates to $11 \pm 1 \mathrm{~nm}$ when alcohol is added [18]. Octanol is a particularly interesting additive because, on the one hand, it is hardly soluble in water $(<1 \%)$ and, on the other hand, $\mathrm{C} 8 \mathrm{O} 3$ molecules are not soluble in octanol preventing segregation. It influences the aggregates' morphology in a similar manner as methanol, only lower concentrations of octanol are needed in order to achieve similar spectral changes [18]. This happens due to its insolubility in water, which ensures that all octanol molecules stay near the aggregate-water interface. Polyvinyl alcohol (PVA) [15] causes dismantling of superhelical assemblies into separate tubular strands. Because of the large size of the PVA molecule and the relatively small inner channel of the single double-wall tubule, which is in the order of $2 \mathrm{~nm}$, only hydrogen bonding assisted adsorption of PVA at the outer surface of the aggregates is possible. Cryo-TEM studies reveal that the tubular morphology is not changed by the presence of PVA except for a slight increase in diameter of the tubule [15]. The anionic surfactant sodium dodecyl sulfate (SDS) added to a C8O3/water solution initially induces formation of single-walled tubules, which in the course of several days are twisted into thick multilamellar tubes [17]. All these modifications in the morphology of the aggregates influence the optical properties of the aggregates by means of the number, positions, and relative strength of absorption bands.

Recently we have demonstrated that linear dichroism (LD) is a powerful tool for characterization of optical properties of C8O3-based aggregates in solution [15, 19]. LD provides information not only on spectral locations of different transitions but also on the orientation of their transition dipole moments. Details on methodology of LD and polarized absorption experiments as well as on the preparation of $\mathrm{C} 8 \mathrm{~S} 3$ and $\mathrm{C} 8 \mathrm{O} 3$ aggregates are described in detail in $[15,19]$. As one can see from Figure 2 where polarized absorption and LD spectra of various TBC-based aggregates are shown, all aggregates feature a multiple band absorption spectrum (bands are numbered from 1 to 4 starting from low energy side) that is red shifted with respect to the absorption 


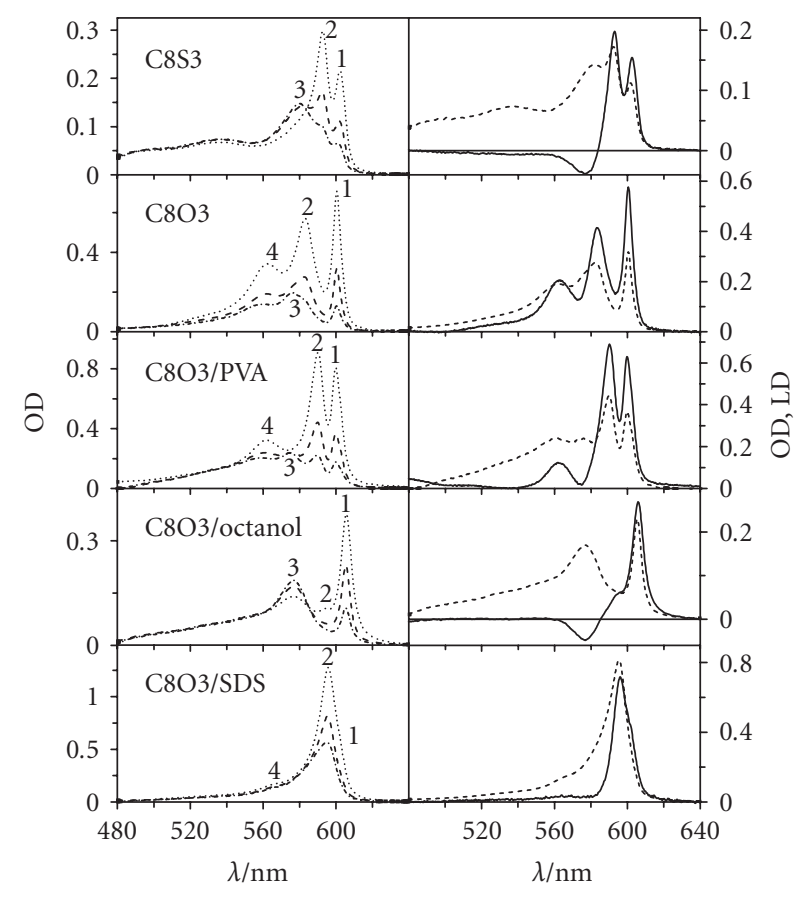

FIgURE 2: Polarized absorption and linear dichroism spectra of various TBC-based aggregates. The left panels show the absorption spectra measured with light polarized parallel (dotted line) and perpendicular (dash-dotted line) to the flow direction; the dashed line shows the isotropic spectrum. The absorption bands are numbered from 1 to 4 starting at low-energy side. The right panels show the corresponding linear dichroism spectra (solid line), together with the isotropic absorption spectrum (dashed line) for comparison.

of single TBC molecule, which has it's maximum at around $520 \mathrm{~nm}$. This red shift evidences a dominating J-type interaction between the transition dipole moments of neighboring $\mathrm{TBC}$ chromophores in the aggregate. In the case of $\mathrm{C} 8 \mathrm{~S} 3 \mathrm{ag}$ gregates two additional bands are found on the blue side of the spectrum which overlaps with the absorption spectrum of a monomer solution. For the various $\mathrm{C} 8 \mathrm{O} 3$ aggregates a residual absorption on the blue side of the spectrum is also present. In this case, however, there is no correspondence to the monomer spectrum, suggesting the presence of weakly allowed transitions to the higher lying states of one-exciton band.

A common feature for all TBC-based cylindrical aggregates is the lowest energy absorption band (1) situated in the vicinity of $600 \mathrm{~nm}$ and representing a parallel polarized transition. For pure $\mathrm{C} 8 \mathrm{O} 3$ and $\mathrm{C} 8 \mathrm{O} 3 / \mathrm{PVA}$ and $\mathrm{C} 8 \mathrm{O} 3 / \mathrm{SDS}$ aggregates it has a maximum at $600 \mathrm{~nm}$ while with addition of short chain alcohols it shifts by a few nanometers to the red and broadens. In the case of C8S3 aggregates this band has its maximum at $605 \mathrm{~nm}$.

The position and intensity of band 2, which also represents transitions oriented along the aggregate axis, is much more sensitive to the presence of different additives. In the case of pure $\mathrm{C} 8 \mathrm{O} 3$ aggregates (bundles of double-wall tubules) band 2 is centered at $583 \mathrm{~nm}$. For isolated double- wall tubules (C8O3/PVA [15], and pure C8S3 [19]) the strong band 2 absorption is centered near $590 \mathrm{~nm}$. The fact that PVA, a large polymer molecule, acts only on absorption band 2 suggests that this band originates from transitions in outer wall of the double-wall tubule.

In the case of $\mathrm{C} 8 \mathrm{O} 3 /$ short-chain alcohol samples, a direct, rapid decay of the $583 \mathrm{~nm}$ has been observed, while a new band is formed at $590 \mathrm{~nm}$. The $590 \mathrm{~nm}$ band vanishes with storage time of the sample. Intuitively this could be explained by a rather rapid dismantling of the bundles directly after addition of alcohol. Indeed cryo-TEM measurements reveal the existence of separate double-wall tubules in the solution even after a few days of storage of the sample [17] which is followed by a slow formation of a new type of thick fiber-like bundles. Consequently, the $590 \mathrm{~nm}$ transition can be referred to as a characteristic of an isolated double-wall tubule. This idea is supported by the fact that pure C8S3 aggregates, which undergo a slow transition from a singletubule to a bundle-like structure, feature spectral changes which are roughly opposite to the ones observed for $\mathrm{C} 8 \mathrm{O} 3$ upon addition of PVA [15] or SDS [17]. Namely, with storage of C8S3 aggregates the $590 \mathrm{~nm}$ band diminishes while the absorption around $580 \mathrm{~nm}$ as well as the absorption of the $600 \mathrm{~nm}$ band increases.

All investigated aggregates feature a band 3 located in the spectral region $575-580 \mathrm{~nm}$. These transitions have dipole moments oriented perpendicular to the direction of alignment. The band is present both in the case of aggregates having a single strand as well as in those having bundle morphology. Furthermore, polarized absorption spectra indicate that the position of this perpendicular transition is to some extent correlated to the position of band 1 .

Finally, absorption band 4, which is only observed in $\mathrm{C} 8 \mathrm{O} 3$ samples and is caused by transitions oriented parallel to the aggregate axis, is very sensitive to environment. In the case of the C8O3/PVA samples, where separate doublewall tubules are present, the band is quite pronounced, while in the case of $\mathrm{C} 8 \mathrm{O} 3 / \mathrm{SDS}$ aggregates which are also characterized as separate double-wall tubules [17] the band is absent. The $560 \mathrm{~nm}$ band is also not present in the case of C8O3/alcohol solutions where big bundles or even fibers of double-wall tubules are formed. Furthermore, the LD in the vicinity of $560 \mathrm{~nm}$ in the case of addition of short chain alcohols becomes negative with increasing storage time. This strongly suggests that $560 \mathrm{~nm}$ band is not related to the interaction between double-wall tubules but instead to a different packing of molecules within the tubule arising from the modified environment. The sensitivity of the transition energies and intensities to slightest changes in arrangement of $\mathrm{C} 8 \mathrm{~S} 3$ molecules in the aggregate has recently been demonstrated by Didraga et al. [19].

The excitonic character of the transitions determining the absorption spectra of TBC-based cylindrical aggregates is confirmed by frequency-resolved pump-probe spectroscopy [20, 21]. Pump-probe spectra measured at zero delay between the pump and probe pulses for different excitation wavelengths corresponding to different absorption bands of pure $\mathrm{C} 8 \mathrm{O} 3$ aggregates are shown in Figure 3. 




FIgURE 3: Zero-delay pump-probe spectra of pure C8O3 aggregates at excitation wavelengths $600 \mathrm{~nm}$ (dash-dotted line), $580 \mathrm{~nm}$ (dashed line), and $560 \mathrm{~nm}$ (solid line). Polarization of the probe beam is parallel to the flow. The dotted line represents the isotropic linear absorption spectrum.

Experimental details of frequency-resolved polarization selective pump-probe experiments are given in [20]. The energy of the excitation pulse was $3 \mathrm{~nJ}$. Experiments with excitation pulse energies down to $10 \mathrm{pJ}$ revealed no changes in the shape of the pump-probe spectra. In the case of excitation at $600 \mathrm{~nm}$ (dash-dotted line) the pump-probe spectrum consists of one photoinduced absorption band and one bleaching-stimulated emission band centered around $600 \mathrm{~nm}$, that is, in the vicinity of the lowest energy absorption band 1. The shape of the pump-probe spectrum is typical for one-dimensional J-aggregates with the photoinduced absorption band blue shifted with respect to the bleaching/stimulated emission band [22] and is caused by the quasifermionic nature of the exciton. A similar shape of the pump-probe spectrum was predicted theoretically [23] and confirmed experimentally [21] for cylindrical J-aggregates as well.

When exciting at $580 \mathrm{~nm}$ (band 2) the zero-delay pumpprobe spectrum (dashed line in Figure 3 ) is dominated by bleaching/stimulated emission and photoinduced absorption bands, which are blue shifted and situated near $580 \mathrm{~nm}$, that is, in the vicinity of absorption band 2 of the aggregates. This clearly demonstrates that the zero-delay pump-probe spectrum in this case is partially caused by a different exciton system than in the case of $600 \mathrm{~nm}$ excitation. The presence of optical density changes at around $600 \mathrm{~nm}$ in the spectrum can be explained either by very fast energy transfer between these excitonic manifolds, or by insufficient spectral selectivity of the excitation pulse. Note that bands 1,2, and 3 largely overlap, which makes truly selective excitation hardly possible.

We emphasize that while exciting at $600 \mathrm{~nm}$, no optical density changes are observed around $580 \mathrm{~nm}$. This means that the excitons manifested at around $600 \mathrm{~nm}$ and $580 \mathrm{~nm}$

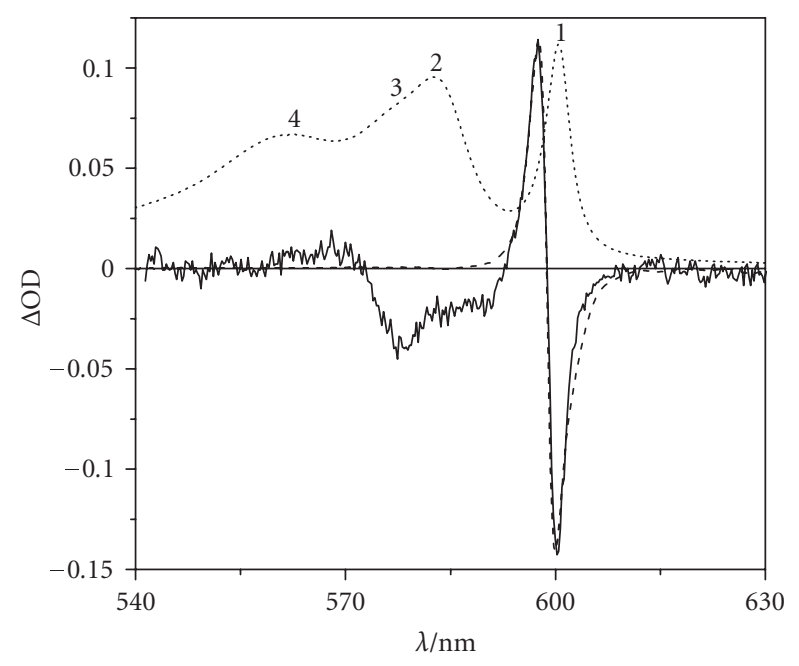

FIgURE 4: Scaled zero-delay pump-probe spectra of pure $\mathrm{C} 8 \mathrm{O} 3 \mathrm{ag}$ gregates at $600 \mathrm{~nm}$ excitation, with the polarization of the probe beam parallel (dashed line), and perpendicular (solid line) to the flow. The dotted line shows the isotropic linear absorption spectrum for comparison.

have different ground states or, in other words, are only weakly coupled. Otherwise, a bleaching at around $580 \mathrm{~nm}$ should be induced instantaneously while exciting at $600 \mathrm{~nm}$. By taking into account the double-wall geometry of the cylindrical aggregates this finding can be interpreted as follows. First, the excitons manifesting themselves in the vicinity of the absorption bands 1 and 2 are located on the separate walls of the double-wall cylinder, as it was implied by LD measurements in the case of pure $\mathrm{C} 8 \mathrm{O} 3$ and $\mathrm{C} 8 \mathrm{O} 3 / \mathrm{PVA}$ aggregates, and second, the interaction between the two walls of the cylinder is small.

In the case of excitation at around $560 \mathrm{~nm}$ (solid line) only a small photobleaching is induced around $560 \mathrm{~nm}$, that is, at the wavelength corresponding to the most blue absorption band (4) of the aggregate. The largest instantaneous photoinduced optical density changes are observed in the vicinity of the bands 1 and 2. Despite the relatively strong linear absorption at $560 \mathrm{~nm}$ the photoinduced optical density changes are small compared to the cases of excitation at $580 \mathrm{~nm}$ and $600 \mathrm{~nm}$. Furthermore, even with the better spectral selectivity of the excitation, $\triangle \mathrm{OD}$ is clearly present in the entire pump-probe spectrum of the absorption bands 1,2 , and 4 directly after excitation. This is an indication that transitions corresponding to the absorption band 4 on the one hand and bands 1 and 2 on the other are to a large extent associated, that is, excitons located on different walls of the aggregate have allowed transitions in the vicinity of $560 \mathrm{~nm}$.

Figure 4 shows zero-delay pump-probe spectra measured for polarizations perpendicular and parallel to the aggregates (to the flow) using $600 \mathrm{~nm}$ excitation. As one can see, in the case of perpendicular polarization a relatively strong bleaching around $578 \mathrm{~nm}$, that is, in the vicinity of the absorption band 3, is induced. This is in addition to the earlier discussed OD changes around $600 \mathrm{~nm}$. Photo-bleaching 
induced at an energy higher than that of the excitation reveals that both transitions situated around $600 \mathrm{~nm}$ and transitions situated around $578 \mathrm{~nm}$ share the same ground state. Again, this strongly suggests that both transitions are located on the same wall of the double-wall cylinder.

Zero-delay pump-probe spectra of C8O3/PVA and S8S3 aggregates are analogous to the ones obtained for the pure $\mathrm{C} 8 \mathrm{O} 3$ samples with bleaching and photoinduced absorption bands situated at wavelengths relevant to the absorption bands in linear absorption spectrum.

\section{EXCITON DYNAMICS}

Exciton dynamics in $\mathrm{C} 8 \mathrm{O} 3$ molecular aggregates was recently studied by means of frequency-resolved polarization selective pump-probe experiments [24]. Here we concentrate on time- and frequency-resolved fluorescence as well as fluorescence quantum yield (QY) measurements in C8O3 aggregates and monomers. Fluorescence involves the one-exciton to ground state transition only, and is a straightforward method to study exciton dynamics.

Time-resolved fluorescence measurements were performed on solutions of $\mathrm{C} 8 \mathrm{O} 3$ that were flowed through a fused silica cell of $0.1 \mathrm{~mm}$ thickness at a rate of about $5 \mathrm{~mL} / \mathrm{s}$. Fluorescence decays were measured by using either a streak camera with a time resolution of 7 picoseconds, or a fluorescence upconversion setup with a typical time resolution of $\sim 200$ femtoseconds. In order to detect spectrally resolved fluorescence decay, however, the gate beam was spectrally limited to $2 \mathrm{~nm}$ by use of a grating, two lenses, and a slit placed in between the lenses. This reduced the time resolution of the experiments to about 2 picoseconds.

In the case of the streak camera measurements, the sample was irradiated by the frequency-doubled output (495 nm) of a tunable $80 \mathrm{MHz}$ Ti: Sapphire laser (Mira 900, Coherent). A pulse picker was used to reduce the repetition rate to $1.9 \mathrm{MHz}$. In the fluorescence upconversion measurements, the sample was excited with 30 femtoseconds pulses at $560 \mathrm{~nm}$ by a signal wave of a noncollinearly pumped optical parametric amplifier (TOPAS WHITE, Light Conversion ltd.). The excitation pulses with energies that were varied between $10 \mathrm{pJ}$ and $15 \mathrm{~nJ}$ were focused onto the sample by a $10 \mathrm{~cm}$ lens.

Fluorescence spectra of pure $\mathrm{C} 8 \mathrm{O} 3$ aggregates and monomers together with the isotropic absorption spectra are plotted in Figure 5. The fluorescence spectrum of $\mathrm{C} 8 \mathrm{O} 3 \mathrm{ag}-$ gregates at room temperature consists of two bands which fully overlap with the two lower energy absorption bands 1 and 2. Their intensity ratio nicely follows the Boltzmann law, implying that at room temperature thermal equilibrium is eventually achieved on a time scale much shorter than the fluorescence radiative lifetime [25].

The fluorescence quantum yield of $\mathrm{C} 8 \mathrm{O} 3$ monomers in ethanol and that of $\mathrm{C} 8 \mathrm{O} 3$ aggregates in $10^{-2} \mathrm{M} \mathrm{NaOH}$ water solution are determined by comparison with Rhodamine $6 \mathrm{G}$ in ethanol $(\Phi=0.95[26])$ and is found to be $0.085 \pm 0.02$ $(8.5 \%)$ and $0.035 \pm 0.01(3.5 \%)$ for the monomers and aggregates, respectively.

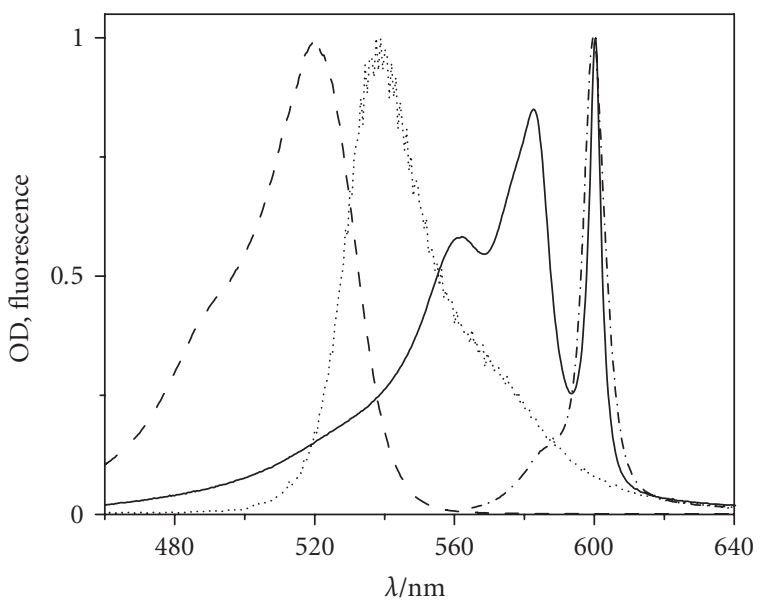

FIGURE 5: Normalized absorption and fluorescence spectra of C8O3 monomers (absorption-dashed line; fluorescence-dotted line), and aggregates (absorption—solid line; fluorescence-dash-dotted line).

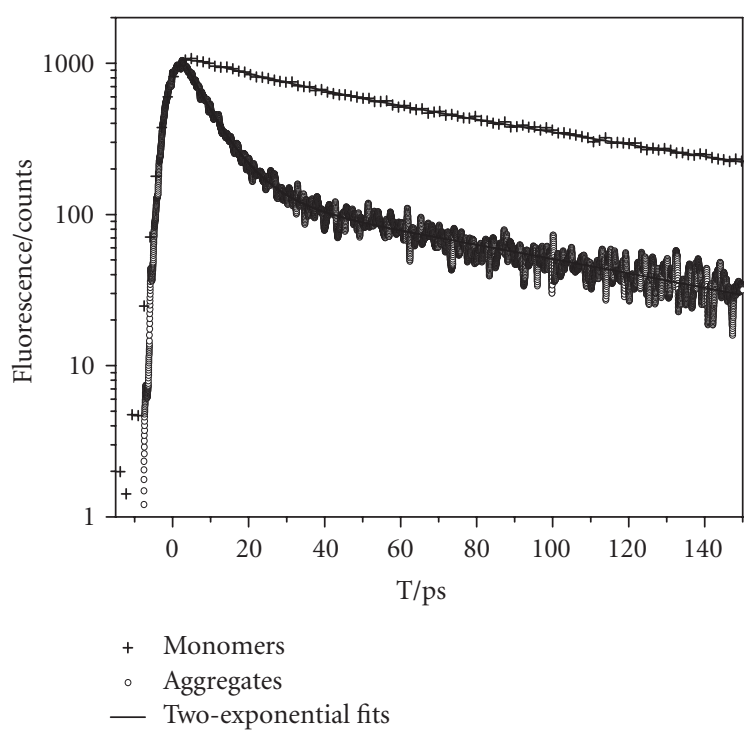

Figure 6: Fluorescence decay of $\mathrm{C} 8 \mathrm{O} 3$ monomers (crosses) and aggregates (circles) as detected with a streak camera. Solid lines represent two-exponential fits (for details see text).

Time-resolved fluorescence measurements (Figure 6) reveal that the fluorescence of $\mathrm{C} 8 \mathrm{O} 3$ monomers dissolved in methanol decays two exponentially with time constants of 30 picoseconds (relative weight $30 \%$ ) and 115 picoseconds. At present, the origin of this two-exponential decay of monomers of $\mathrm{C} 8 \mathrm{O} 3$ derivatives, and of the 30 picoseconds decay component is unclear. The fluorescence decay of $\mathrm{C} 8 \mathrm{O} 3$ aggregates also shows a two-exponential decay. The decay rate is substantially increased yielding time constants of 5 picoseconds (relative weight $\sim 93 \%$ ) and 105 picoseconds. No fluorescence spectral dynamics is observed at room temperature within the 7 picoseconds streak camera time resolution. 
Despite the deconvolution procedure applied, the precision of the determination of the 5 picoseconds decay constant is quite poor. In order to verify the obtained results fluorescence up-conversion experiments were performed. They show (data are presented in Figure 7) that the fluorescence at $605 \mathrm{~nm}$ of the $\mathrm{C} 8 \mathrm{O} 3$ aggregates decays with a 5.5 picoseconds decay time constant.

Using the fluorescence QY and the weight-averaged fluorescence decay time, the radiative lifetime $\tau_{\mathrm{rad}}=\left(\mathrm{QY} / \tau_{\mathrm{FL}}\right)^{-1}$ can be estimated to be 1.2 nanosecond and 285 picoseconds for $\mathrm{C} 8 \mathrm{O} 3$ monomers and aggregates, respectively. Collective effects such as superradiance can explain the fact that the radiative lifetime decreases upon aggregation. On the other hand, the reduced fluorescence QY indicates that additional nonradiative decay channels are opened when $\mathrm{C} 8 \mathrm{O} 3$ molecules self organize into aggregates.

The observed fluorescence decay does not depend on the excitation pulse energy up to $10 \mathrm{pJ}$. By taking into account the irradiated volume $\left(\pi \times(30 \mu \mathrm{m})^{2} \times 1.4 \mathrm{~mm}\right)$ and concentration of $\mathrm{C} 8 \mathrm{O} 3$ molecules $\left(10^{-4} \mathrm{M}\right)$ an excitation energy of $10 \mathrm{pJ}$ corresponds to approximately one photon absorbed per 12.000 molecules. According to cryo-TEM data the physical size of the cylindrical aggregates is about $10 \mathrm{~nm}$ in diameter and on average $300 \mathrm{~nm}$ in length. They therefore contain approximately 10.000 monomer molecules. Consequently, an excitation density of one photon absorbed per 12.000 monomer units implies that on average less than one molecule per aggregate is excited. This explains the absence of features hinting toward exciton-exciton annihilation, that is, the independence of the fluorescence decay time on excitation density.

The time constants of 5.5 picoseconds and 105 picoseconds as measured by monitoring fluorescence dynamics are also characteristic for the decay of the pump-probe signal. Additional dynamics of the pump-probe spectrum on a subpicosecond time scale are observed in the case of excitation at $580 \mathrm{~nm}$ (band 2) (Figure 8). A rapid decay at around $580 \mathrm{~nm}$ (vicinity of band 2) and delayed formation of the spectrum located at around $600 \mathrm{~nm}$ (band 1) are observed. Analysis of the pump-probe transients (Figure 9) gives a single time constant of $275 \pm 10$ femtoseconds for both the subpicosecond decay and the formation which points towards a precursor-successor relation implying rather fast energy transfer (ET) from the outer to the inner tubule. The kinetics shown in Figure 9 was fitted three exponentially with time constants 275 femtoseconds, 5 picoseconds, and 105 picoseconds. Given the weak interaction between the $\mathrm{C} 8 \mathrm{O} 3$ molecules located in the inner and outer tubules, as discussed above, and an energy transfer time constant of 275 femtoseconds, which is more than an order of magnitude slower than the dephasing time at room temperature [14], an incoherent energy transfer, characterized by Förster's theory can be suggested. The decay of the signal at $578 \mathrm{~nm}$, corresponding to the exciton located on the outer tubule, is independent of the excitation intensity up to quite high excitation pulse energies reaching $1.5 \mathrm{~nJ}$. This corresponds to one photon absorbed per circa 100 monomer molecules. It strongly suggests that the

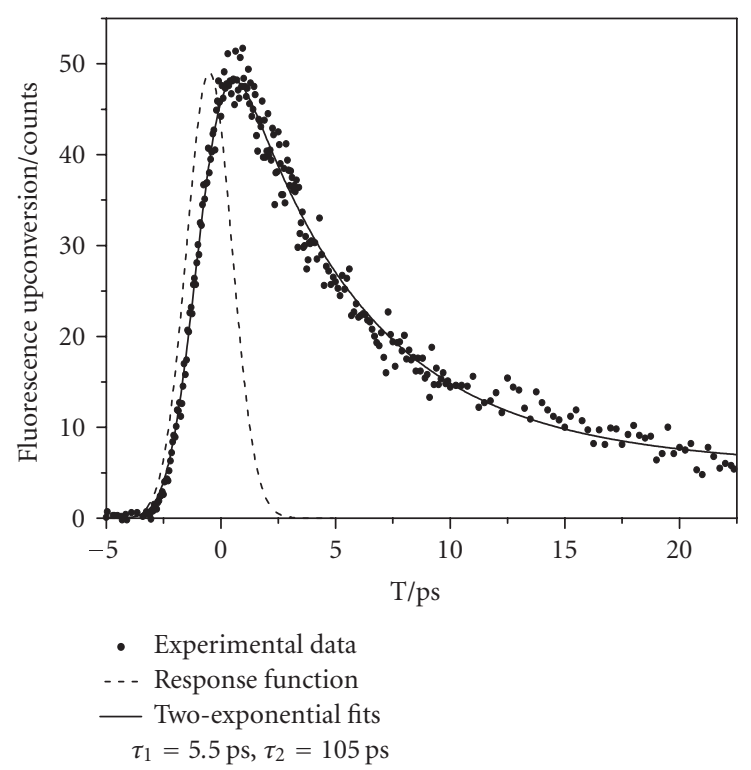

Figure 7: Initial fluorescence decay in $\mathrm{C} 8 \mathrm{O} 3$ aggregates as detected by fluorescence upconversion (dots). Solid line: two-exponential fit with time constants 5.5 and 105 picoseconds. The response function is given by the dashed line.

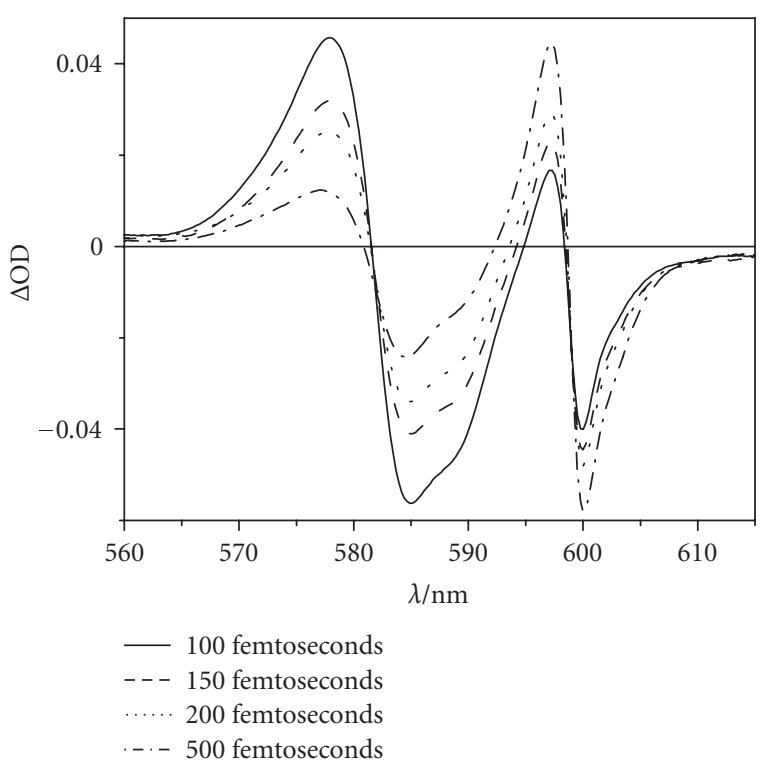

FIGURE 8: Dynamics of the pump-probe spectrum of pure C8O3 aggregates during the first 500 femtoseconds after excitation at $580 \mathrm{~nm}$.

exciton delocalization length does not exceed this number of molecules.

When PVA is added to the solution, the "donor" band 2 shifts about $7 \mathrm{~nm}$ towards the "acceptor" band 1. The Förster model predicts faster energy transfer when the spectral overlap increases. Instead, it is observed that the ET time constant increases to $450 \pm 20$ femtoseconds. This is probably 


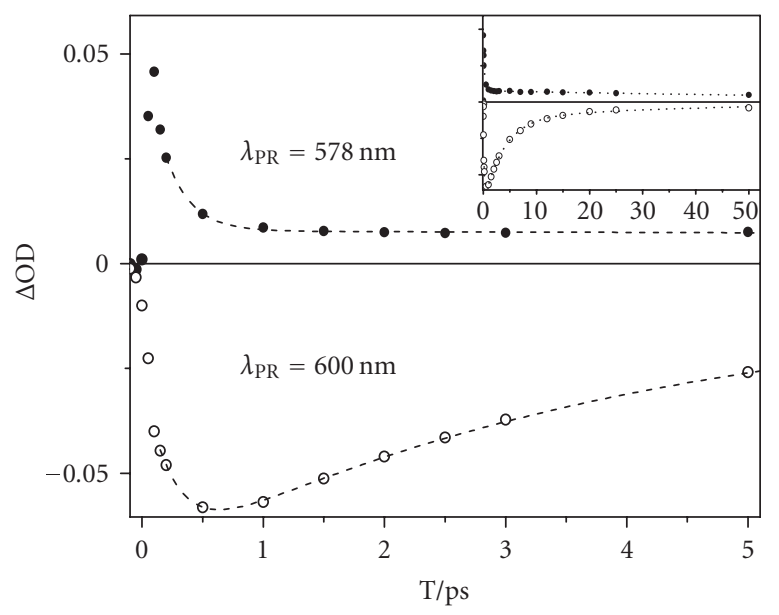

FIgURE 9: Decay of pump-probe transients during the first 5 picoseconds following excitation measured at probe wavelengths $600 \mathrm{~nm}$ (open circles) and $578 \mathrm{~nm}$ (filled circles), which corresponds to the bleaching/stimulated emission of band 1 and to the photoinduced absorption in the vicinity of band 2, respectively. In the insert, the dynamics on a 50 picoseconds timescale are shown. Dashed lines represent three-exponential fits with time constants 275 femtoseconds, 5.5 picoseconds, and 105 picoseconds (see text for details).

the result of an increase of the distance between both walls of the cylinder. Indeed, cryogenic electron transmission microscopy shows that the diameter of the cylinders increases from $10 \pm 1 \mathrm{~nm}$ to $11.5 \pm 1 \mathrm{~nm}$ upon addition of PVA. The energy transfer rate thus provides detailed information on the microscopic structure.

The dynamics of the pump-probe spectrum during the first 20 picoseconds after excitation at $600 \mathrm{~nm}$ is shown in Figure 10. The right panel shows an expanded view of the dynamics in the $565-590 \mathrm{~nm}$ spectral region. The excitation of the lowest energy exciton is followed by the usual decay, but also by a matched growth of the pump-probe spectrum around $580 \mathrm{~nm}$. This precursor-successor relation reveals an energy transfer from the inner to the outer wall of the double-wall tubule. The energy transfer rate from the lower to the higher energy exciton was measured to be intensity dependent. It speeds up with increasing excitation density, and shows a distinct nonexponential behavior at higher excitation densities. Most probably exciton migration makes it possible for two excitons to interact and thereby populate a two-exciton state, which in turn contributes to the energy transfer process between the inner and outer walls.

At low excitation density ( 1 absorbed photon per 10.000 monomer molecules) the transfer dynamics from the low energy to the high energy exciton takes place with a time constant of $3.5 \pm 2$ picoseconds. The relatively large error in the time constant is caused by a rather small increase of the signal around $585 \mathrm{~nm}$ at low excitation density. Furthermore, the obtained time constant can be compared to the short fluorescence lifetime in $\mathrm{C} 8 \mathrm{O} 3$ aggregates $(5.5 \pm 1$ picoseconds), making a determination of the energy transfer time constant inaccurate.

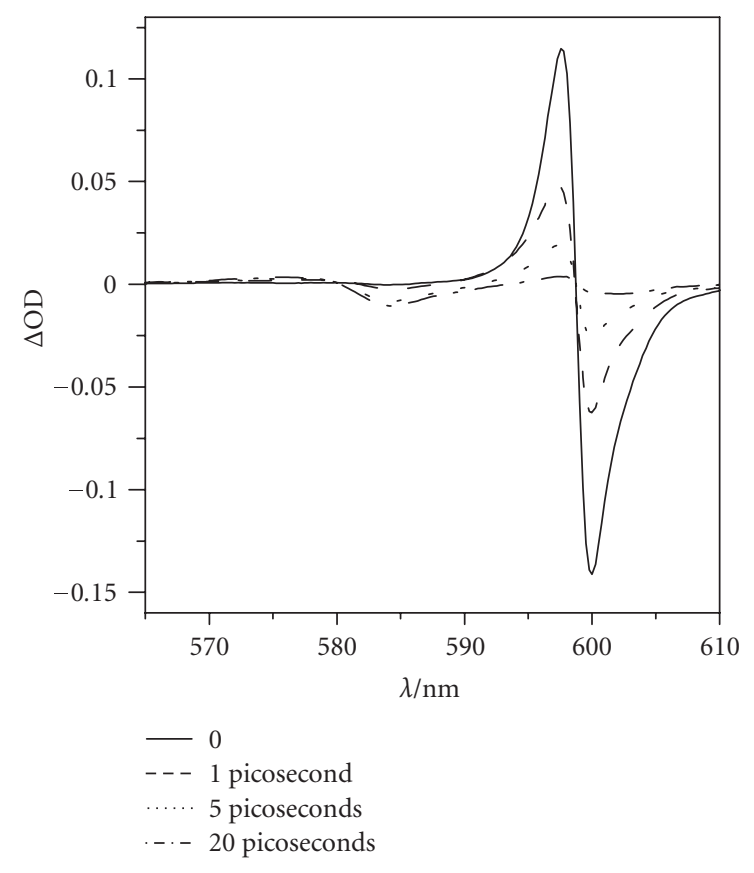

(a)

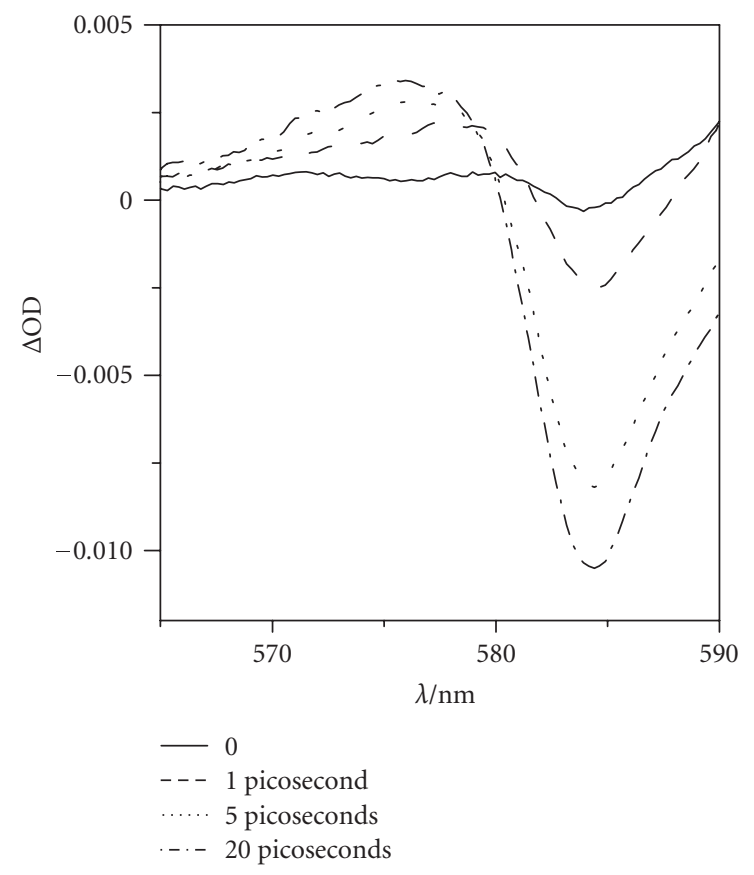

(b)

Figure 10: (a) Dynamics of the pump-probe spectrum of pure $\mathrm{C} 8 \mathrm{O} 3$ aggregates during the first 20 picoseconds after excitation at $600 \mathrm{~nm}$, (b) dynamics in the vicinity of $580 \mathrm{~nm}$ (band 2).

\section{CONCLUSIONS}

The optical properties and exciton dynamics of doublewalled cylindrical aggregates of carbocyanine dyes have been discussed. Linear dichroism measurements on oriented samples revealed that the absorption spectra of such cylindrical structures consist of a set of relatively narrow and strongly polarized bands. The morphology and spectral properties of 
the substituted 5,5',6,6'-tetrachlorobenzimidacarbocyanine aggregates depend on the particular side groups attached as well as possible additives. Within the class of tubular aggregates, however, generic features seem to exist, in particular the occurrence of two lowest-energy J-bands polarized parallel to the cylinder axis and one higher-energy band polarized perpendicular to it. Exact positions and strengths of these transitions are influenced by the choice of side groups and solvent. This suggests that all of these aggregates have the same basic structure, even though the precise values of the structural parameters may vary. Spectral changes occurring upon addition of PVA suggest that the lowest-energy band originates from excitations on the inner wall of the cylinder.

The absorption spectra of pure $\mathrm{C} 8 \mathrm{O} 3$ and C8O3/PVA aggregates feature a fourth (parallel) band at higher energy $(\sim 560 \mathrm{~nm})$ that is currently unexplained. However, timeresolved pump-probe and fluorescence studies shine some light on its nature. The zero-delay pump-probe spectrum first reveals that the two parallel, lowest-energy excitons (at 600 and $583 \mathrm{~nm}$, resp.) possess different ground states; this confirms the idea that the excitations are on different walls of the tubule. Furthermore, the time dependence of the pumpprobe signal shows that energy transfer occurs between the walls with a time constant of circa 275 femtoseconds. Therefore the inner and outer walls of the tubule must be weakly coupled. Finally, when the unaccounted-for $563 \mathrm{~nm}$ parallel band is excited, ground-state bleaching is observed within the overlap of the pump and probe at both the positions of the inner-wall and outer-wall exciton manifolds. From this we conclude that both exciton manifolds contribute to the oscillator strength at $560 \mathrm{~nm}$.

Time-resolved fluorescence measurements reveal substantial speeding of fluorescence decay with aggregation. Considering the fluorescence QY and weight-averaged fluorescence decay time, radiative lifetimes of 1.2 nanosecond and 285 picoseconds are found for $\mathrm{C} 8 \mathrm{O} 3$ monomers and aggregates, respectively. Collective effects such as superradiance can explain the fact that the radiative lifetime decreases upon aggregation. On the other hand, a reduced fluorescence QY indicates that additional nonradiative decay channels are opened when $\mathrm{C} 8 \mathrm{O} 3$ molecules self organize into aggregates.

Finally uphill energy transfer (i.e., from the "600 nm" to the " $583 \mathrm{~nm}$ " exciton band) is observed on a picosecond timescale. Strong dependence of the energy transfer on the excitation density points towards an annihilation-assisted process. Observed increase of the energy transfer rate may result from direct transitions from two-exciton states as well as from a local temperature increase as a result of excitation annihilation.

\section{ACKNOWLEDGMENTS}

This work is part of the research program of the "Stichting voor Fundamenteel Onderzoek der Materie (FOM)," which is financially supported by the "Nederlandse Organisatie voor Wetenschappelijk Onderzoek (NWO)." The authors acknowledge Dr. H. von Berlepsch from the Freie Universität
Berlin and Dr. S. Kirstein from the Humbolt Universität zu Berlin for valuable discussions on morphology-related optical properties of $\mathrm{C} 8 \mathrm{O} 3$ aggregates. The authors also gratefully acknowledge close and ongoing collaboration with the Institute of Theoretical Physics of Groningen University and in particular with Professor J. Knoester, Dr. V. Malyshev, and Dr. C. Didraga.

\section{REFERENCES}

[1] G. Scheibe, "Über die Veränderlichkeit des Absorptionsspektrums einiger Sensibilisierungsfarbstoffe und deren Ursache," Angewandte Chemie, vol. 49, p. 563, 1936.

[2] G. Scheibe, "Über die Veränderlichkeit der Absorptionsspektren in Lösungen und die Nebenvalenzen als ihre Ursache," Angewandte Chemie, vol. 50, no. 11, pp. 212-219, 1937.

[3] E. E. Jelley, "Spectral absortion and fluorescence of dyes in the molecular state," Nature (London), vol. 138, p. 1009, 1936.

[4] E. E. Jelley, "Molecular, nematic and crystal states of 1,1Diethyl-c-cyaninechloride," Nature (London), vol. 139, p. 631, 1937.

[5] A. Mishra, R. K. Behera, P. K. Behera, B. K. Mishra, and G. B. Behera, "Cyanines during the 1990s: a review," Chemical Reviews, vol. 100, no. 6, pp. 1973-2011, 2000.

[6] F. Würthner, R. Wortmann, and K. Meerholz, "Chromophore design for photorefractive organic materials," ChemPhysChem, vol. 3, no. 1, pp. 17-31, 2002.

[7] T. Kobayashi, J-Aggregates, World Scientific, Singapore, Singapore, 1996.

[8] V. I. Prokhorenko, D. B. Steensgaard, and A. R. Holzwarth, "Exciton dynamics in the chlorosomal antennae of the green bacteria Chloroflexus aurantiacus and Chlorobium tepidum," Biophysical Journal, vol. 79, no. 4, pp. 2105-2120, 2000.

[9] S. Karrasch, P. A. Bullough, and R. Ghosh, "The $8.5 \AA$ A projection map of the light-harvesting complex I from Rhodospirillum rubrum reveals a ring composed of 16 subunits," EMBO Journal, vol. 14, no. 4, pp. 631-638, 1995.

[10] G. McDermott, S. M. Prince, A. A. Freer, et al., "Crystal structure of an integral membrane light-harvesting complex from photosynthetic bacteria," Nature, vol. 374, no. 6522, pp. 517521, 1995.

[11] A. Pawlik, A. Ouart, S. Kirstein, H.-W. Abraham, and S. Dähne, "Synthesis and UV/Vis spectra of $J$-aggregating $5,5^{\prime}, 6,6^{\prime}$-tetrachlorobenzimidacarbocyanine dyes for artificial light-harvesting systems and for asymmetrical generation of supramolecular helices," European Journal of Organic Chemistry, no. 16, pp. 3065-3080, 2003.

[12] H. Von Berlepsch, C. Böttcher, A. Ouart, C. Burger, S. Dähne, and S. Kirstein, "Supramolecular structures of J-aggregates of carbocyanine dyes in solution," Journal of Physical Chemistry $B$, vol. 104, no. 22, pp. 5255-5262, 2000.

[13] C. Spitz and S. Dähne, "Architecture of J-aggregates studied by pressure-dependent absorption and fluorescence measurements," Berichte der Bunsengesellschaft/Physical Chemistry, Chemical Physics, vol. 102, no. 5, pp. 738-744, 1998.

[14] M. van Burgel, D. A. Wiersma, and K. Duppen, "The dynamics of one-dimensional excitons in liquids," The Journal of Chemical Physics, vol. 102, no. 1, pp. 20-33, 1995.

[15] H. Von Berlepsch, S. Kirstein, P. R. Hania, C. Didraga, A. Pugzlys, and C. Böttcher, "Stabilization of individual tubular $J$ aggregates by poly(vinyl alcohol)," Journal of Physical Chemistry B, vol. 107, no. 51, pp. 14176-14184, 2003. 
[16] H. Von Berlepsch, M. Regenbrecht, S. Dähne, S. Kirstein, and C. Böttcher, "Surfactant-induced separation of stacked Jaggregates. Cryo-transmission electron microscopy studies reveal bilayer ribbons," Langmuir, vol. 18, no. 7, pp. 2901-2907, 2002.

[17] H. Von Berlepsch, C. Böttcher, A. Ouart, et al., "Surfactantinduced changes of morphology of J-aggregates: superhelixto-tubule transformation," Langmuir, vol. 16, no. 14, pp. 5908-5916, 2000.

[18] H. Von Berlepsch, S. Kirstein, and C. Böttcher, "Effect of alcohols on J-aggregation of a carbocyanine dye," Langmuir, vol. 18, no. 20, pp. 7699-7705, 2002.

[19] C. Didraga, A. Pugzlys, P. R. Hania, H. Von Berlepsch, K. Duppen, and J. Knoester, "Structure, spectroscopy, and microscopic model of tubular carbocyanine dye aggregates," Journal of Physical Chemistry B, vol. 108, no. 39, pp. 14976-14985, 2004.

[20] A. Pugzlys, P. R. Hania, C. Didraga, J. Knoester, and K. Duppen, "Cylindrical aggregates of TDBC: linear and nonlinear optical properties versus morphology," Diffusion and Defect Data Pt.B: Solid State Phenomena, vol. 97-98, pp. 201-206, 2004.

[21] S. S. Lampoura, C. Spitz, S. Dähne, J. Knoester, and K. Duppen, "The optical dynamics of excitons in cylindrical $J$ aggregates," Journal of Physical Chemistry B, vol. 106, no. 12, pp. 3103-3111, 2002.

[22] H. Fidder, J. Knoester, and D. A. Wiersma, "Observation of the one-exciton to two-exciton transition in a $J$ aggregate," The Journal of Chemical Physics, vol. 98, no. 8, pp. 6564-6566, 1993.

[23] M. Bednarz and J. Knoester, "The linear absorption and pump-probe spectra of cylindrical molecular aggregates," Journal of Physical Chemistry B, vol. 105, no. 51, pp. 1291312923, 2001.

[24] A. Pugzlys, P. R. Hania, C. Didraga, V. Malyshev, J. Knoester, and K. Duppen, "Ultrafast exciton transport in organic nanotubes," Springer Series in Chemical Physics, vol. 79, pp. 879$881,2004$.

[25] P. R. Hania, In Ultrafast Laser and Spectroscopy Laboratory, pp. 155, University of Groningen, Groningen, The Netherlands 2005.

[26] R. F. Kubin and A. N. Fletcher, "Fluorescence quantum yields of some rhodamine dyes," Journal of Luminescence, vol. 27, no. 4, pp. 455-462, 1982. 


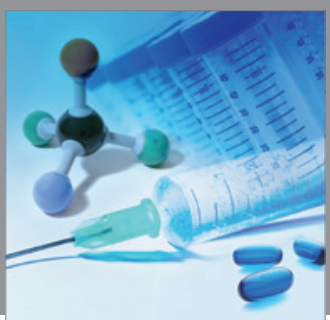

International Journal of

Medicinal Chemistry

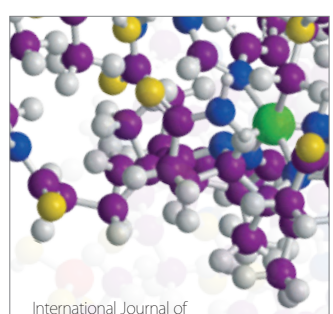

Carbohydrate Chemistry

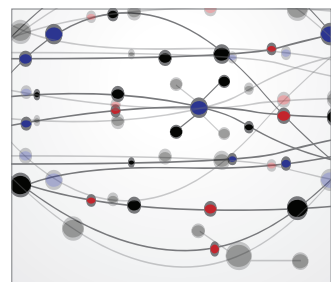

The Scientific World Journal
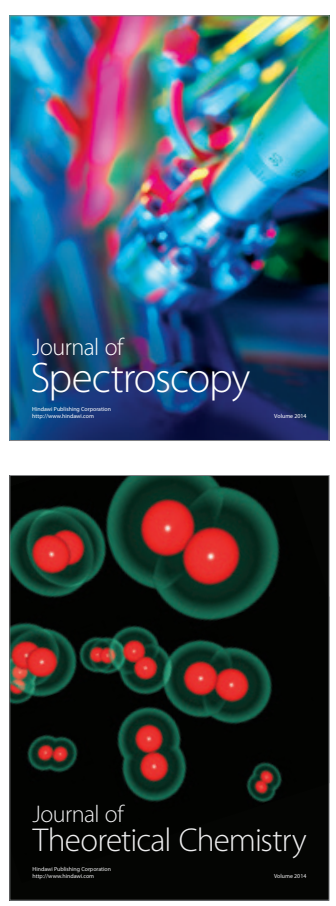
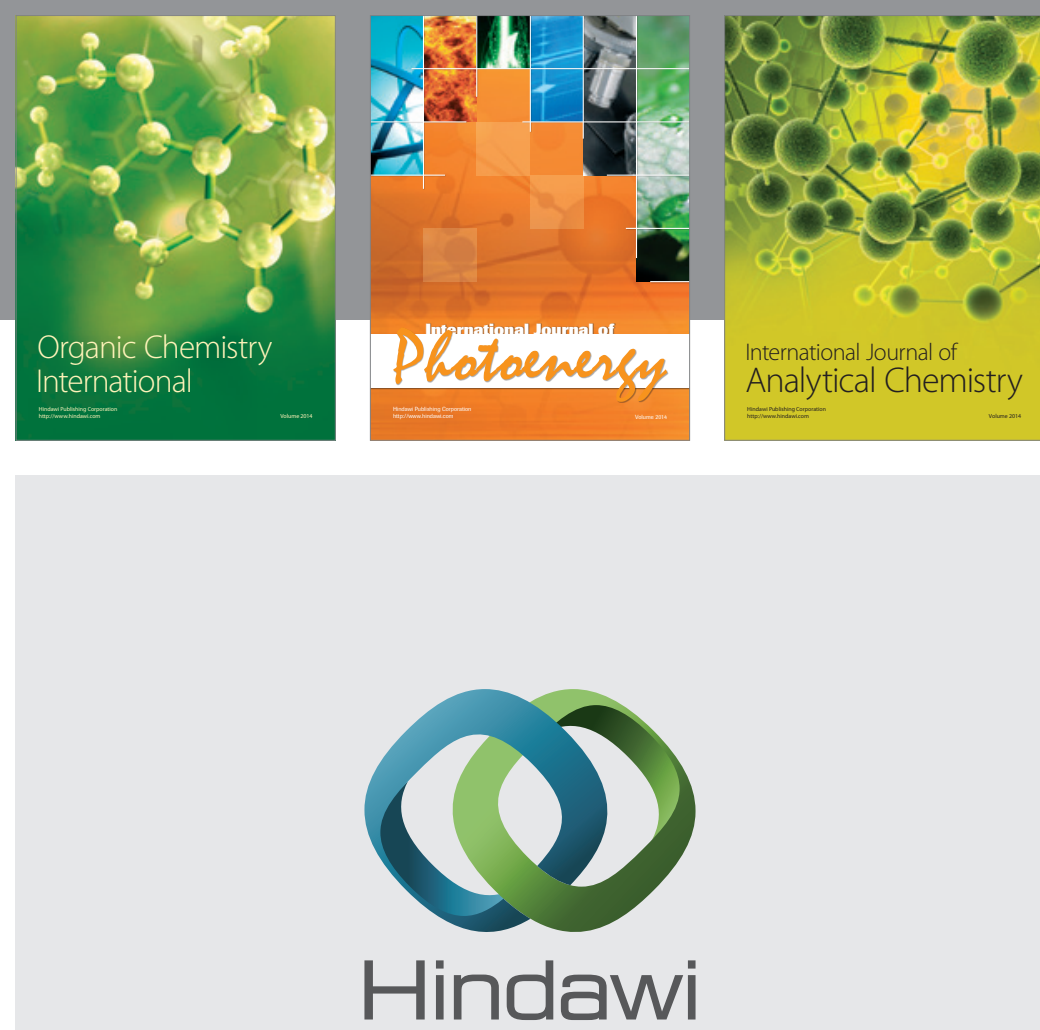

Submit your manuscripts at

http://www.hindawi.com
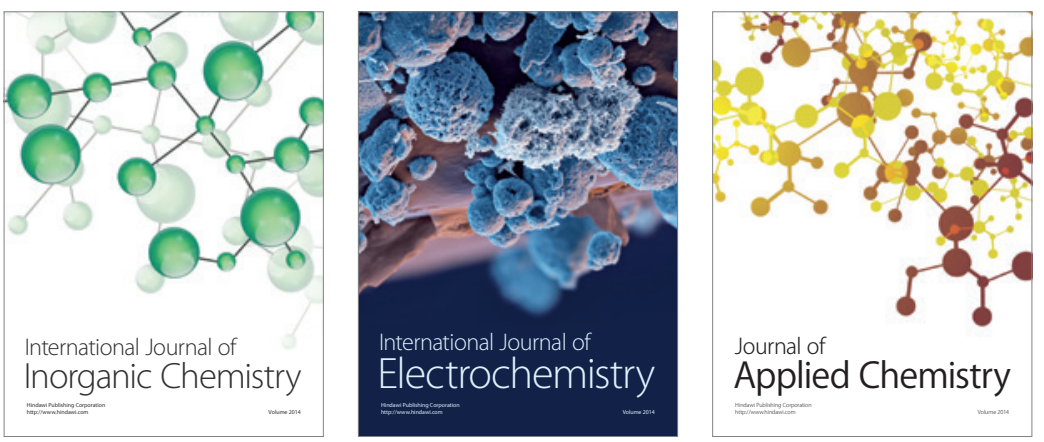

Journal of

Applied Chemistry
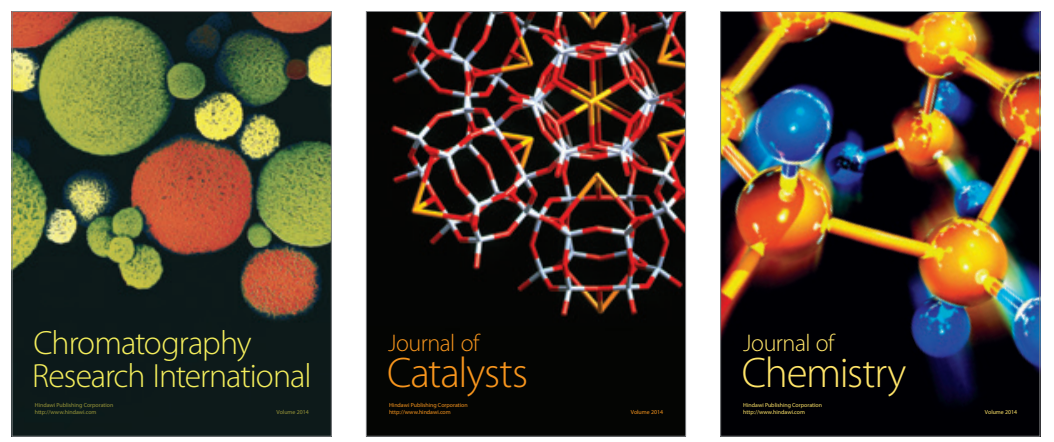
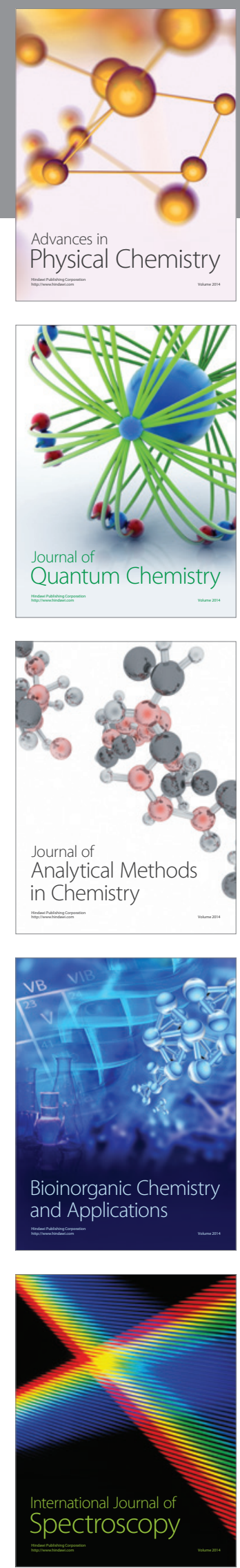